\title{
DEFORMATION RETRACTS AND WEAK DEFORMATION RETRACTS OF NONCOMPACT MANIFOLDS ${ }^{1}$
}

\author{
GUDRUN KALMBACH
}

1. Introduction. This note gives a proof of the following result: Let $M$ be either a smooth noncompact manifold with a countable base or a compact manifold with nonempty boundary. Let $f$ be a nondegenerate function on $M$ with a finite number of critical points $p_{1}, \cdots, p_{r}$ with indices $\lambda_{1}, \cdots, \lambda_{r}$ resp., where in the noncompact case $f\left(q_{i}\right) \rightarrow \infty$ for each sequence $q_{i} \in M$ without limit point or in the compact case $f(p)=a$ on the boundary points $p$ for some fixed $a \in R$. If $E_{i}$ is the open descending $\lambda_{i}$-dimensional bowl, determined by $p_{i}$, [4], and $K=\mathrm{U}_{i=1,2}, \ldots E_{i}$, then $K$ is a deformation retract of $M$.

Functions with an infinite number of critical points on $M$ do not have this property in general: there exists a simple example of a twodimensional manifold $M$ and a nondegenerate function $f$ on $M$ with an infinite number of critical points of index 1 , a single critical point of index 0 and no critical point of index 2 , such that $K$ is not a deformation retract of $M$.

But the theorem above holds in such a case in the form that $K$ is a "weak deformation retract," (see $\S 4$ ), of $M$.

2. Notation and preliminary notes. The notation defined in this paragraph will be used throughout this note.

$M=M^{n}$ is a smooth, noncompact manifold of dimension $n$ with a countable base.

$f$ is a nondegenerate function on $M,[1]$, with critical points

$p_{1}, p_{2}, p_{3}, \cdots$ and $f\left(p_{1}\right)<f\left(p_{2}\right)<f\left(p_{3}\right)<\cdots$ and with $f\left(q_{k}\right) \rightarrow \infty$ if the sequence $q_{k} \in M$ has no limit point in $M$.

$\lambda_{i}$ is the index of $f$ at $p_{i}$.

$E_{i}$ is the descending $\lambda_{i}$-bowl associated with $p_{i},[4$, p. 83]

$D_{M}$ is the set of smooth functions on $M$.

$\Delta f(p)$ is the gradient of $f$ at $p$.

Presented to the Society, January 26, 1968 under the title Some remarks on noncompact manifolds; received by the editors October 26, 1967.

1 This is a part of the author's dissertation, Über niederdimensionale CW-Komplexe in nicht-kompakten Mannigfaltigkeiten, Göttingen, 1966, thesis adviser: Professor H. Grauert.

The writer thanks Professor S. S. Cairns for discussion and suggestions.

This report was prepared under grant NSF GP 5610. 
For each noncritical point $p$ of $M$, (that is, for each point $p$ where $\Delta f(p)$ is not zero), there is an orthogonal trajectory to the level surfaces of $f$ through $p$.

$\phi(p)$ denotes the (maximal) orthogonal trajectory through $p$ to the level surfaces of $f$ such that $\phi(p)$ contains as subsets all other orthogonal trajectories of $f$ through $p$; we write also

$\phi$ for the set $\{\phi(p) \mid p \in M\}$. (If $p$ is a critical point of $f$ then we set $\phi(p)=\{p\}$.

$K=\underset{i=1,2, \cdots}{\bigcup} E_{i}, \quad M^{a}=\{p \in M \mid f(p) \leqq a\}, \quad \partial M^{a}=\{p \in M \mid f(p)=a\}$.

It is possible to choose the Riemannian metric on $M$ such that for each point $p_{i}$ there exists a neighborhood $U_{i}$ in $M$ with coordinate functions $x$ in $U_{i}$ such that $f(x)$ is given in $U_{i}$ by

$$
f(x)=-\sum_{k=1}^{\lambda_{i}} x_{k}^{2}+\sum_{k=\lambda_{i}+1}^{n} x_{k}^{2}+f\left(p_{i}\right)
$$

and the system of differential equations of $\phi$ in $U_{i}$ is given by

$$
\begin{array}{ll}
d z_{k}(t) / d t=-2 z_{k} & \text { for } k=1, \cdots, \lambda_{i}, \\
d z_{k}(t) / d t=2 z_{k} & \text { for } k=\lambda_{i}+1, \cdots, n .
\end{array}
$$

Then $\phi$ is locally given by $\phi(t, x)=\left(e^{-2 t} x_{1}, \cdots, e^{-2 t} x_{\lambda_{i}}, e^{2 t} x_{\lambda_{i}+1}, \ldots\right.$, $\left.e^{2 t} x_{n}\right)$. Let $A \subset M$ and $B \subset M$ be closed sets in $M$ with $A, B \neq \varnothing$ and $A \cap B=\not \varnothing$. A function $g \in D_{M}$ is called a separation-function of $A$ and $B$ in $M$ if $g(p)=0$ for $p \in A$ and $g(p)=1$ for $p \in B$, if $0 \leqq g(p) \leqq 1$ in $M$ and $\Delta g(p) \neq 0$ for all points $p \in M$ with $0<g(p)<1$.

REMARK 1. If $U \subset M$ is open and $h \in D_{u}$; if $a<b$ and $B$ $=\{p \in U \mid h(p) \leqq a\} \neq \varnothing$, if $A=U-\{p \in U \mid h(p)<b\} \neq \varnothing$ and the gradient $\Delta h$ of $h$ is $\neq 00$ on $\{p \in U \mid a \leqq h(p) \leqq b\}=C$, then there exists a separation-function $g$ of $A$ and $B$ in $U$ such that $\Delta g(p) \neq 0$ in Int $(C)$ and the level manifolds of $g$ on $C$ are the same as the level manifolds of $h$.

If one takes the function

$$
G(y)=\frac{\int_{\nu}^{b} \exp [-1 /(t-a)+1 /(t-b)] d t}{\int_{a}^{b} \exp [-1 /(t-a)+1 /(t-b)] d t}
$$

for real numbers $y$ with $a \leqq y \leqq b$ and $G(y)=0$ if $y \geqq b$, respectively $G(y)=1$ if $y \leqq a$; if we take $g(p)=G(h(p))$ then all conditions of the remark are fulfilled.

$g_{a, b, h}=g$ always means the function $g$ of this remark, it depends on the numbers $a, b$ and the function $h$. 
Remark 2. Let $U \subset M$ be open and $h \in D_{\text {u. }}$ Let

$$
G=\{p \in U \mid h(p)=d\}
$$

with $d \in R$, let $\Delta h(p) \neq 0$ if $p \in G$. Then the set $\chi$ of maximal orthogonal trajectories $\chi(p)$ of $h,(p \in G)$, is defined in $U$ and for suitable parameters of $\chi$ in $N=\{q \in \chi(p) \mid p \in G\}$ and suitable neighborhoods $U_{1}$ and $U_{2}$ of a point $p \in G$ and $\chi_{\imath}(p) \in N$ respectively the correspondence

$$
x_{1}:\left\{\begin{array}{c}
U_{1} \rightarrow U_{2} \\
p \rightarrow x_{t}(p)
\end{array}\right\}
$$

is a diffeomorphism ([1, p. 13], $t$ is fixed). Therefore, if $F \in D_{G}$ and $q=\chi_{t}(p)$, then $g(q)=g\left(\chi_{t}(p)\right)=F(p),(p \in G)$, defines a function $g$ in $D_{N}$ and if $\Delta F(p) \neq 0$ for all $p \in G$ then $\Delta g(q) \leftarrow 0$ for all $q \in N$.

REMARK 3. $\bar{E}_{i} \subset K$.

Proof. Let $q_{i}$ be a sequence of points in $E_{i}$ which has as limit point $p$ some point of $M$ not in $E_{i}$, then it may happen that $p=p_{k}$, is a critical point; in which case $p \in K$. If not, then only a finite number of points $q_{i}$ can be contained in a maximal orthogonal trajectory of $f$, that means that there exists an infinite subsequence $q_{1}^{\prime}, q_{2}^{\prime}, \cdots$ of $q_{i}$ with $\phi\left(q_{m}^{\prime}\right) \cap \phi\left(q_{e}^{\prime}\right)=\varnothing$ if $m \neq e$. These orthogonal trajectories converge in a neighborhood of $p$ to the orthogonal trajectory $\phi(p)$ and in $U_{i}$ (a neighborhood of $p_{i}$ ) to an orthogonal trajectory $g \subset E_{i}$, [2]. It follows that $\phi(p) \cap g=\phi$ since $p \notin E_{i}$, so $\phi(p)$ has a critical point $p_{k}$ as a boundary point in $M-M^{f(p)}$, so $p \in \phi(p) \subset E_{k} \subset K$.

3. Special vector fields on subsets of $M$. Let $\epsilon_{i}>0$ be a suitable constant,

$$
V_{i}^{\prime \prime}=\left\{x \in U_{i} \mid h_{i}^{\prime \prime}(x)=\sum_{j=\lambda_{i}+1}^{n} x_{j}^{2}<\epsilon_{i}\right\}
$$

and

$$
V_{i}^{\prime}=\left\{p \in \phi(x) \cap M^{f(x)} \mid x \in V_{i}^{\prime \prime}\right\} .
$$

Proposition 1. Let $\lambda_{i}<n$. There exists a function $h_{i} \in D_{V i^{\prime}}$ such that $\Delta h_{\boldsymbol{i}}(p)=0$ if $p \in E_{\boldsymbol{i}}$ and $\Delta h_{\boldsymbol{i}}(p) \neq 0$ if $p \in V_{\boldsymbol{i}}^{\prime}-E_{\boldsymbol{i}}$.

Proof. Take $a^{\prime}<b^{\prime}<f\left(p_{i}\right)$ with $\left(\partial M^{a^{\prime}}\right) \cap V_{i}^{\prime} \subset U_{i}$ and $g(p)$ $=g_{a^{\prime}, b^{\prime}, f}(p)$ if $p \in V_{i}^{\prime}$. If $x \in U_{i}$ and $x^{\prime}=\phi(x) \cap \partial M^{a^{\prime}}$, then

$$
x^{\prime}=\left(e^{-2 t} x_{1}, \cdots, e^{-2 t} x_{\lambda_{i}}, e^{2 t} x_{\lambda_{i}+1}, \ldots, e^{2 t} x_{n}\right)
$$

for some $t \in R$ (see $\S 2$ ). The function $h^{+}(p)=h_{i}^{\prime \prime}\left(\phi_{t}(p)\right)$ with $\phi_{t}(p)$ 
$=\phi(p) \cap \partial M^{a^{\prime}} \cap V_{i}^{\prime}$ is defined in $V_{i}^{\prime}-\left\{p_{i}\right\}$ according to Remark 2. It follows that $0 \leqq h^{+}(x)=e^{4 t} h_{i}^{\prime \prime}(x) \leqq h_{i}^{\prime \prime}(x)$ if $t \leqq 0$ and $x \in U_{i}$. Define $h_{i}(p)=(1-g(p)) h_{i}^{\prime \prime}(p)+g(p) h^{+}(p)$. If $x \in U_{i} \cap\left(M^{b^{\prime}}+\operatorname{Int}\left(M^{a^{\prime}}\right)\right)$ and $x \notin E_{i}$ then

$$
\Delta f\left(h_{i}(x)\right)=(1-g(x)) \Delta f\left(h_{i}^{\prime \prime}(x)\right)+\left(-h_{i}^{\prime \prime}(x)+h^{+}(x)\right) \Delta f(g(x))>0
$$

so $\Delta h_{i}(x) \neq 0$. If $x \in E_{i} \cap U_{i}$ then $\Delta h_{i}(x)=0$. It follows from the preceding two sentences and from the definition of $h_{i}$ that $\Delta h_{i}(p)=0$ if $p \in E_{i}$, otherwise $\Delta h_{i}(p) \neq 0$ in $V_{i}^{\prime}$ and that $\Delta h_{i}(p)$ is tangent to the level surface $\partial M^{c}$ if $p \in \partial M^{c} \cap V_{i}^{\prime}-U_{i}$.

Some notation. Let $0<2 \epsilon_{i}^{\prime}<\epsilon_{i}$ and $g_{i}(p)=g_{\epsilon i, 2 \epsilon i, h_{i}}^{\prime}(p)$ if $p \in V_{i}^{\prime}$ $(\operatorname{Remark} 1) ;$ let $W_{i}^{\prime}=\left\{p \in V_{i}^{\prime} \mid g_{i}(p)=0\right\}$ and $W_{i}=\left\{p \in V_{i}^{\prime} \mid g_{i}(p)=1\right\}$. We take an arbitrary noncritical value $c$ of $f$ and the set $K^{c}=\bigcup_{i: p_{i} \in M^{c}} E_{i}$ and we will show that $K^{c}$ is a deformation retract of $M^{c}$. We take first exactly the critical points $p_{i_{1}}, p_{i_{2}}, \cdots, p_{i_{\text {d }}}$ with the properties $p_{i_{v}} \in M^{c}$ and $\lambda_{i_{v}}<n$. Let $V_{e}=\bigcup_{v=e}^{s} V_{i_{v}}^{\prime}, 1 \leqq e \leqq s$; let $U_{e}^{\prime}=\bigcup_{v=e}^{s}, W_{i_{v}}$, $U_{e}^{+}=\bigcup_{v=e}^{s}\left(V_{i_{v}}^{\prime}-W_{i_{v}}^{\prime}\right), B^{e}=\bigcup_{v=e}^{s} E_{i_{v}}$ and $U^{c}=\bigcup_{v=1}^{s} U_{i_{v}}$.

Proposition 2. There exists a separation function $g_{k}^{\prime}$ of $V_{k}-U_{k}^{+}$and $U_{k}^{\prime}$ in $V_{k}$.

Proof. If $k=s$ then such a function exists, namely $g_{a}^{\prime}=g_{i,}$. For some $k \leqq s$ assume that the function $g_{k}{ }^{\prime}$ exists, then

$$
g_{k-1}^{\prime}(p)=1-\left(1-g_{k}^{\prime}(p)\right)\left(1-g_{i_{k-1}}(p)\right)
$$

has the desired properties for $k-1$ instead of $k$.

Proposition 3. If there exists a number $k$ with $1 \leqq k \leqq s$ and $a$ vector field $X_{k}$ on $U_{k}^{\prime}$ such that $X_{k}(p)=0$ if $p \in B^{k}$ and $X_{k}(p) \neq 0$ if $p \in U_{k}^{\prime}-B^{k}$ and $X_{k}(p)$ is tangent to the level surface $\partial M^{a}$ if $p \in \partial M^{a} \cap\left(U_{k}^{\prime}-B^{k} \cup U^{c}\right)$ with $a \in R$, then there exists a vector field $X_{k-1}$ on $U_{k-1}^{\prime}$ with the properties of $X_{k}$ mentioned above (where $k$ is replaced by $k-1$ ).

Proof. We take $X_{k-1}^{\prime}(p)=g_{k}^{\prime}(p) X_{k}(p)+\left(1-g_{k}^{\prime}(p)\right) \Delta h_{i_{k-1}}(p)$ in $V_{k-1}^{\prime}-E_{i_{k-1}}$. Without restriction, $X_{k}(f(p))=0$ if $p \in V_{k-1}^{\prime} \cap V_{k}$. We have

$$
\begin{aligned}
X_{k-1}^{\prime}(f(p))=\left(1-g_{k}^{\prime}(p)\right) \Delta h_{i_{k-1}}(f(p)) & \geqq 0 \\
& >0
\end{aligned}
$$

if $\left(1-g_{k}^{\prime}(p)\right) \neq 0$ and $\Delta h_{i_{k-1}}(f(p)) \neq 0$. This is the case if $a^{\prime}, h^{\prime \prime}$ are taken as in Proposition 1 if $h_{i_{k-1}}^{\prime \prime}(p)>0$ and $f(p)>a^{\prime}$ i.e., if $p \in U_{i_{k-1}}$ $-E_{i_{k-1}}$ with $f(p)>a^{\prime}$ and $\left(1-g_{k}^{\prime}(p)\right) \neq 0$. Therefore $X_{k-1}^{\prime}(p) \neq 0$ in $V_{k-1}^{\prime}-B^{k-1}$. Take $g^{\prime}(p)=1-g_{0, \epsilon_{k-1}, h_{i_{k-1}}^{\prime \prime}}(p)$ with $0<\epsilon_{k-1}^{\prime \prime}<\epsilon_{i_{k-1}}^{\prime}$ and 
$X_{k-1}(p)=g^{\prime}(p) X_{k-1}^{\prime}(p)$. Then $X_{k-1}$ is a smooth vector field on $V_{k-1}^{\prime}$ $\cap U_{k-1}^{\prime}$ since $g^{\prime} \cdot g_{k}^{\prime} \in D_{V_{k-1}^{\prime}}^{\prime}$. The vector field $X_{k-1}$ has the desired properties.

Proposition 4. Let $V^{c}=U_{1}^{\prime} \cup K^{c}$. Then $K^{c}$ is a deformation retract of $V^{c}$.

Proof. Proposition 1 gives a vector field $X_{s}(p)=\Delta h_{i_{s}}(p)$ with the properties of Proposition 3 and $k=s$. So Proposition 3 gives a vector field $X_{1}$ on $U_{1}^{\prime}$ with the properties of $X_{k}$ (with $k=1$ ). $V^{c}$ is a neighborhood of $K^{c}$ in $M^{c}$. To each point $p \in V^{c}-K^{c}$ there exists a maximal trajectory $\chi(p)$ of $X_{1}$ through $p$ and $\chi(p)$ has exactly one limit point $p^{\prime} \in K^{c}$. Let $d_{p}$ be the length of the segment of $\chi(p)$ between $p$ and $p^{\prime}$. If $p \in K^{c}$ we define $d_{p}=0$ and $\chi(p)=\{p\}$. Then $d_{p}$ is continuous. The correspondence $p \rightarrow q \in \chi(p)$ with $d_{q}=(1-t) d_{p}$ and $0 \leqq t \leqq 1$ gives a deformation retract of $V^{c}$ on $K^{c}$.

4. Proof of the theorem. Let $K$ be defined as in $\$ 1$ and $K^{c}$ as in $\S 3$. Then we call $K$ a weak deformation retract of $M$, if for each (noncritical) value $c \in R$ the set $K^{c}$ is a deformation retract of $M^{c}$.

Theorem. $K$ is a weak deformation retract of $M$.

Proof. Take $\epsilon_{i}^{\prime}$ and $i_{1}, \cdots, i_{\text {a }}$ as in $\S 3$ and $0<2 a_{i}<\epsilon_{i}^{\prime}$. Let $A$ $=\cup_{i: \lambda_{i}=n_{;} p_{i} \in M^{c}} E_{i}$ and $f_{i}(p)=g_{a_{i}, 2 a_{i}, h_{i}}(p)$ if $p \in V_{i}^{\prime}-A$ and let $g^{+}(p)$ $=1-\prod_{r=1}^{s}\left(1-f_{i_{v}}(p)\right)$. If $p \in A$ or $p \in M^{c}-V^{c}$ then we define $g^{+}(p)=1$ or $g^{+}(p)=0$ respectively. Then $g^{+} \in D_{M^{c}}$ (Remark 3$)$. The sets $B=\left\{p \in M^{c} \mid g^{+}(p)=0\right\}$ and $C=\left\{p \in M^{c} \mid g^{+}(p)=1\right\}$ are disjoint and closed in $M^{c}$, so $g^{+}$is a separation function of $B$ and $C$ in $M^{c}$. Let $X_{1}(p)$ be as in Proposition 4 if $p \in V^{c}-A$ and $X_{1}(p)=0$ if $p \in A$; then the vector field $X^{*}(p)=g^{+}(p) X_{1}(p)+\left(1-g^{+}(p)\right) \Delta f(p)$ is smooth on $M^{c}$. We have $X^{*}(p) \neq 0$ if $g^{+}(p) \neq 1$ since $X_{1}(f(p)) \geqq 0$.

The deformation retract of $M^{c}$ on $K^{c}$ is given in the following way: let $d_{p}$ be the length of the segment of the maximal trajectory $\psi(p)$ of $X^{*}$ between $p$ and $K^{c}$ if $p \in M^{c}-K^{c}$ and let $d_{p}=0$ and $\psi(p)=\{p\}$ if $p \in K^{c}$. Then the correspondence $p \rightarrow q \in \psi(p)$ with $d_{q}=(1-t) d_{p}$ and $0 \leqq t \leqq 1$ gives the desired deformation retract.

CoRollary. If $f$ has only a finite number of critical points on $M$, then $K$ is a deformation retract of $M$. This is also true if $M$ is a manifold with boundary.

The only remark to make is that if we take a noncritical value $c \in R$ such that $\Delta f(p) \neq 0$ for all $p \in M-M^{c}$ then $X^{*}=\Delta f$ in a neighborhood of $\partial M^{c}$ in $M^{c}$, so with the additional definition $X^{*}(p)=\Delta f(p)$ 
if $p \in M-M^{c}$ we can construct the deformation retract as in the proof of the theorem.

\section{REFERENCES}

1. J. Milnor, Morse theory, Ann. of Math. Studies No. 51, Princeton Univ. Press, Princeton, N. J., 1963.

2. P. Montel, Sur l'intégrale superieure et l'intégrale inférieure d'une équation differentielle, Bull. Sci. Math. (2) 50 (1926), 205-217.

3. M. Morse, The existence of polar nondegenerate functions on differentiable manifolds, Ann. of Math. 71 (1960), 352-383.

4. - Bowls of a nondegenerate function on a compact differentiable manifold, Differential and Combinatorial Topology, Princeton Univ. Press, Princeton, N. J., 1965, 81-103.

UNIVERSITY OF ILLINOIS 